\title{
Electrochemical Degradation Characteristics of Refractory Organic Pollutants in Coking Wastewater on Multiwall Carbon Nanotube-Modified Electrode
}

\author{
Yan Wang, Shujing Sun, Guifu Ding, and Hong Wang \\ National Key Laboratory of Nano/Micro Fabrication Technology, Key Laboratory for Thin Film and Microfabrication, \\ Ministry of Education, Shanghai Jiaotong University, Shanghai 200240, China \\ Correspondence should be addressed to Yan Wang, wyyw@sjtu.edu.cn
}

Received 19 May 2011; Accepted 7 August 2011

Academic Editor: Steve Acquah

Copyright ( $) 2012$ Yan Wang et al. This is an open access article distributed under the Creative Commons Attribution License, which permits unrestricted use, distribution, and reproduction in any medium, provided the original work is properly cited.

The multiwall carbon nanotube-mollified electrode (MWCNT-ME) was fabricated and its electrocatalytic activity of refractory organic pollutants of coking wastewater was investigated. The surface morphology, absorption properties, and the electrochemical behavior of phenol and aniline at the MWCNT-ME were analyzed. Using ultraviolet-visible adsorption spectroscopy (UV-vis), Gas chromatography mass spectrometry (GC/MS), and chemical oxygen demand (COD) test, the electrochemical oxidation properties of refractory organic pollutants of coking wastewater using the MWCNT-ME and the IrSnSb/Ti electrode were analyzed. Compared with the powder adsorption media, the MWCNT-ME was proved to have weaker adsorption activity, which means electrochemical degradation is the decisive factor of the removal of organic pollutants. The MWCNT-ME shows high electrochemical reactivity with oxidation peaks of $0.18 \mathrm{~A}$ and $0.12 \mathrm{~A}$ for phenol and aniline, respectively. Under the same working conditions, the MWCNT-ME COD removal rate 51\% is higher than IrSnSb/Ti electrode's rate 35\%. The MWCNT-ME has application potential of electrochemical oxidation of refractory organic pollutants of coking wastewater.

\section{Introduction}

The increasing emission of refractory organic pollutants has challenged the conventional biological treatment. These contaminants such as polynuclear aromatic hydrocarbons PAHs, nitrogen-, oxygen-, and sulfur-containing heterocyclic compounds possess high resistance to microbial degradation or utilization. As one of the advanced treatment systems, electrochemical oxidation has drawn significant attention in water treatment research [1], because of its environmental compatibility, small space requirement, and powerful oxidation ability [2]. The electrode material must have high electrocatalytic activity towards the electrochemical oxidation of organics to $\mathrm{CO}_{2}$ and $\mathrm{H}_{2} \mathrm{O}$ [3]. In addition, this process depends on the activity of coating materials on the electrodes. Recent research has focused on surface modifica- tion of the conventional anodes by nanotube (NT) arrays [4]. The nanoarchitecture of NT arrays features a high surfaceto-volume ratio due to the additional area enclosed inside the hollow structure. As a result of the larger surface area, higher catalytic activity is readily accessible.

With the combination of high aspect ratio, nanometersized dimensions, good electrical conductivity [5], and low capacitance in the pristine state, carbon nanotube film electrodes were used extensively for the electrochemical detection of organic compounds [6] and have potential activity for degradation of organic pollutants [7].

This paper is focused on the electrochemical degradation characteristics of refractory organic pollutants in coking wastewater on a multiwall carbon nanotube-modified electrode (MWCNT-ME). To the best of our knowledge, few studies on electrocatalytic properties of CNT-modified electrodes for degradation of refractory organic pollutants in actual wastewater have been reported. Coking wastewater after biologically purification was chosen as model reactant, in which constituents are low concentration, refractory, toxic, and carcinogenic. The basic electrode configuration of the MWCNT-ME is randomly dispersed multiwall carbon 
nanotubes (MWCNTs) in polytetrafluoroethylene (PTFE) dropped on the surface of a Ti conducting support macroelectrode. Firstly, phenol and aniline were chosen as the model reactants to exam the electrocatalytic characteristics of the MWCNT-ME. As priority pollutants, phenol and aniline are ubiquitous in the environment and have been chosen frequently as typical pollutants because many data are available on their removal and destruction, in particular, with respect to wastewater treatments [8]. Adsorption experiments of organic pollutants were carried out with activated carbon, CNT powder, and MWCNT-ME. The results dedicate that electrochemical oxidation plays the main role for removal of organic compounds. Ultraviolet-visible adsorption spectroscopy (UV-vis), gas chromatography mass spectrometry (GC/MS), and chemical oxygen demand (COD) measurement were carried out to investigate the change of organic compositions in the electrochemical oxidation process. The degrading efficiency of the MWCNT-ME was compared with that of the IrSnSb/Ti electrode which is the widely and typically used dimension-stable anode (DSA).

\section{Experimental Details}

MWCNTs were purchased from Shenzhen Nanotech Port Co., Ltd. (Shenzhen, China), a commercial supplier of carbon nanotubes. MWCNTs have tube diameters of $60-$ $100 \mathrm{~nm}$, lengths of $5-15 \mu \mathrm{m}$, and a purity of more than $95 \%$. PTFE solution with concentration of $30 \%$ (mass percent) was purchased from QINAIRUN Co., Ltd. (Shanghai, China). IrSnSb/Ti electrode was supplied by Suzhou FengGang Co., Ltd. The biologically pretreated coking wastewater was collected from Shanghai Baoshan Iron and Steel Company. Activated carbon, $\mathrm{NaSO}_{4}$, phenol, and aniline were obtained from China National Pharmaceutical Group Corporation (Beijin, China). All the chemicals reagents were of analytical grade and used without further purification. All solutions for voltammeter study were prepared, using doubly distilled water.

2.1. Electrode Preparation. PTFE is a chemically stable material. The fluoropolymer composite film prepared with PTFE and MWCNTs had a high resistance to solvents, acids, and bases [9]. Titanium electrode will be used as the support macroelectrode because it is widely used in the industry of electrochemistry. Titanium meshes $(10 \mathrm{~cm} \times 6 \mathrm{~cm} \times 0.1 \mathrm{~cm}$, $99.5 \%$ purity) whose oxide removed with alkali were rinsed in two $10 \mathrm{~min}$ steps in ultrasonicated acetone and deionized distilled water (DDW) before drying in flowing N2 stream. PTFE solution with concentration of $30 \%$ (mass percent) was used as dispersion fluid. The PTFE solution and MWCNTs were mixed in ethanol with ultrasonic dispersion. The composite dip coated on the prepared titanium mesh and then dried in oven with temperature-programmed mode to $150^{\circ} \mathrm{C}$ for forming the MWCNT-ME $\left(2 \mathrm{~mm}\right.$ thickness, $60 \mathrm{~cm}^{2}$ area).

2.2. Adsorption Experiment. The MWCNTs and activated carbon with same quantity of $1 \mathrm{~g}$ were fed into the two beak-

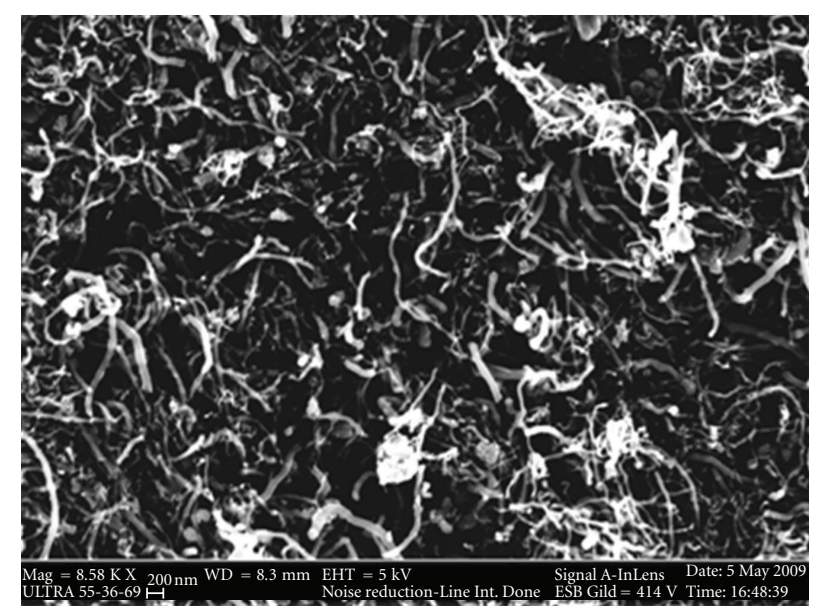

FIGURE 1: The Field-emission SEM (FESEM) top-view image of the MWCNT-ME.

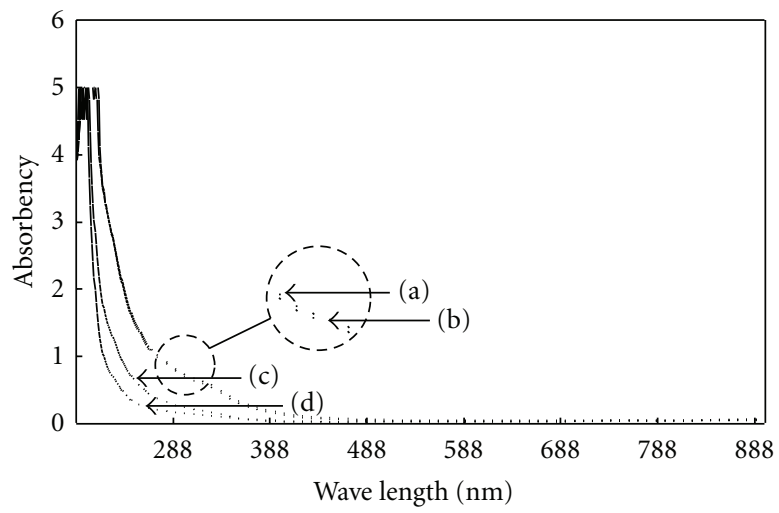

FIGURE 2: UV-Vis spectrum of coking wastewater (a) untreated, (b) treated by the MWCNT-ME, (c) treated by active carbon, and (d) treated by MWCNTs powder.

ers filled with the wastewater with volume of $1 \mathrm{~L}$, respectively. The adsorption experiment lasted for $1 \mathrm{~h}$ and proceeded with magmatic stirring. $5 \mathrm{~mL}$ each of treated wastewater before and after adsorption experiment were taken for UV-vis spectrum test. The same experiment was carried out for the MWCNT-ME which contains $1 \mathrm{~g}$ MWCNTs.

2.3. Electrochemical Measurement. All the electrochemical experiments were performed at $25 \pm 1{ }^{\circ} \mathrm{C}$. The working anodes were tested for their cyclic voltammetry (CV) by CHI-660 electrochemical workstation (Shanghai Chenhua Instrument Company, China), using the CHI version 10.08 software package. The reference electrode is a saturated calomel electrode (SCE). A standard three-electrode configuration was employed along with a cell whose volume was fixed for $250 \mathrm{~mL}$. The acreage of working-electrode-contacting solution was $1 \mathrm{~cm}^{2}$. Distance between working electrode and counter electrode was fixed to be $2 \mathrm{~cm}$. A $200 \mathrm{~mL} \mathrm{Na} \mathrm{SO}_{4}$ $(20 \mathrm{~g} / \mathrm{L})$ solution containing phenol $(0.6 \mathrm{~g} / \mathrm{L})$ and a $200 \mathrm{~mL}$ $\mathrm{Na}_{2} \mathrm{SO}_{4}(20 \mathrm{~g} / \mathrm{L})$ solution containing aniline $(0.6 \mathrm{~g} / \mathrm{L})$ were 


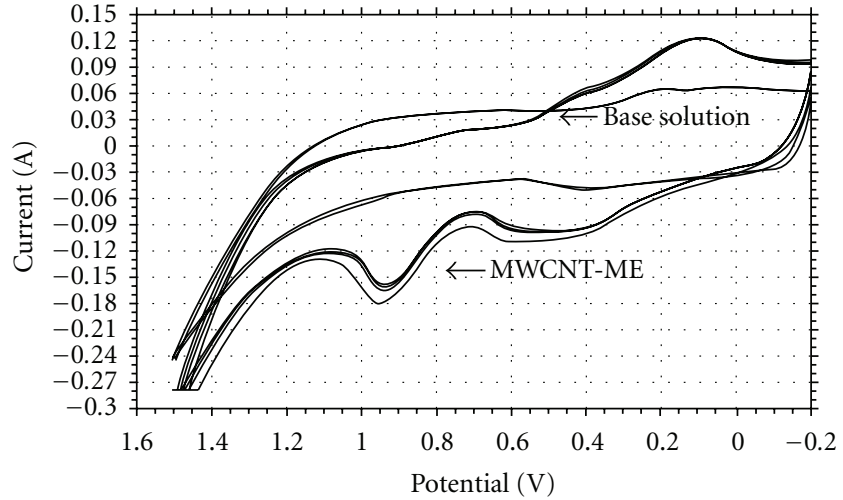

(a)

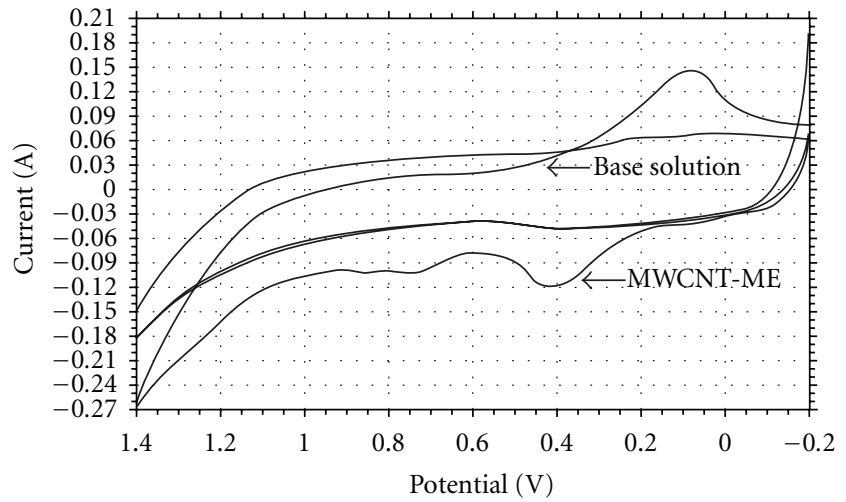

(b)

Figure 3: Performance of MWCNT-ME in oxidation of phenol and aniline, scan rate: $0.01 \mathrm{~V} / \mathrm{s}$ (a) $0.06 \mathrm{~g} / \mathrm{L}$ phenol in $20 \mathrm{~g} / \mathrm{L} \mathrm{NaSO} 4$ and (b) $0.06 \mathrm{~g} / \mathrm{L}$ aniline in $20 \mathrm{~g} / \mathrm{L} \mathrm{NaSO}_{4}$.

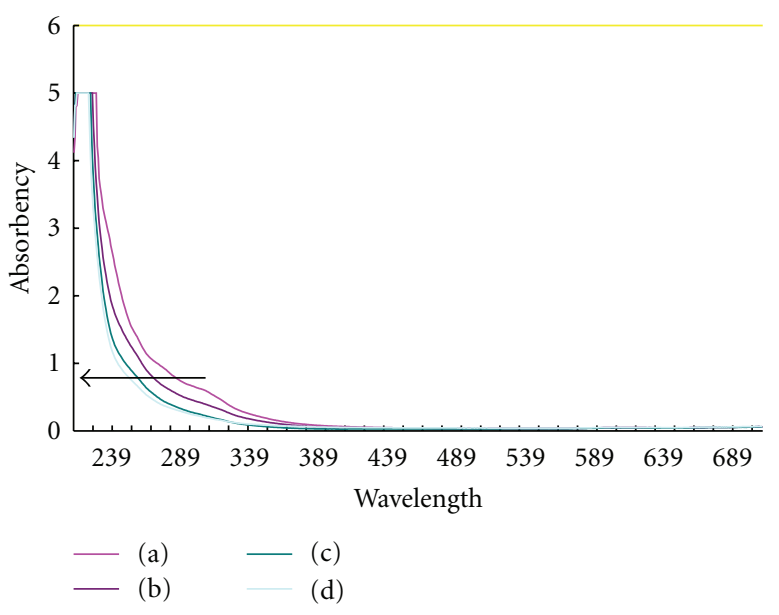

Figure 4: UV-Vis spectra of coking wastewater oxidized by MWCNT-ME for (a) $0 \mathrm{~min}$, (b) $30 \mathrm{~min}$, (c) $60 \mathrm{~min}$, (d) $120 \mathrm{~min}$.

used for test. The electrode was scanned between $-0.2 \mathrm{~V}$ and $1.4 \mathrm{~V}$, the scan rate was $0.01 \mathrm{~V} / \mathrm{S}$.

2.4. Electrochemical Degradation. The degradation experiment of coking wastewater was carried out in an electrochemical cell under magnetic stirring with a volume of $1 \mathrm{~L}$. The performing temperature was at $25 \pm 1^{\circ} \mathrm{C}$. Prior to degradation experiment, coking wastewater was purified biologically and filtered by filter paper to remove solid. The supporting electrolyte was $10 \mathrm{~g} / \mathrm{L} \mathrm{Na}_{2} \mathrm{SO}_{4}$. The MWCNT-ME with wet surface area of $60 \mathrm{~cm}^{2}$ was used as the anode and the platinum sheet with the same wet surface area was used as the cathode. The distance between electrodes was fixed at $5 \mathrm{~mm}$. The applied current density was set at $15 \mathrm{~mA} / \mathrm{cm}^{2}$. The experiment proceeded until total passed charge reached 7200 coulombs. The samples of the wastewater were collected with time interval of $30 \mathrm{~min}$ for UV-vis analysis. The organic constituents of the initial wastewater were analyzed with GC/MS-QP2010 (Shimazu). The same GC/MS tests were carried out for treated wastewater with the MWCNT-ME and the IrSnSb/Ti electrode. The chemical oxygen demand (COD) of wastewater was measured by a titrimetric method using dichromate as the oxidant in acidic solution at $150^{\circ} \mathrm{C}$ for $2 \mathrm{~h}$ (CHEMetrics, USA).

\section{Results and Discussion}

3.1. Morphology of MWCNT-ME. Figure 1 shows a topdown view of representative field-emission SEM image of the MWCNT-ME. As illustrated in Figure 1, MWCNTs with ends and edges exposed on the surface are dispersed homogeneously in PTFE. MWCNTs with mesh-like structure are effectively interwoven around each other and make up an electric mesh with electrochemical sensitive sites on the interface. The dispersed MWCNTs possess high specific volume and large surface area. On the other hand, there exits so many pores forming transition adsorption sites among interwoven MWCNTs, and capillarity provides fast penetration channels in electrochemical degradation process, which enhances the infiltration force of three-phase boundary.

3.2. Adsorption Experiment. As quantum wires made up of curled graphite layers, MWCNTs have obvious adsorption ability for fluid and gas. UV-vis spectrums of samples before and after degradation experiment were shown in Figure 2. It is clear that no distinct adsorption occurring on the MWCNT-ME compared with active carbon and MWCNTs powder.

For single-ring aromatic organic pollutants whose absorbency of UV-vis lies in the range from $200 \mathrm{~nm}$ to $250 \mathrm{~nm}$, active carbon has better adsorption ability than MWCNTs powder. Basic structure cell of the active carbon is graphitelike microcrystalline with hybrid of $\mathrm{sp} 2$, graphite-like microcrystalline cells form the ultrafine particles with nanoscale, and pore structures with different scales can be found in this system in which adsorption can be easy going. For MWCNTs, the adsorption sites lie on inner walls of tubes with open ports and accumulation pores formed among MWCNTs. However, lack of open ports of MWCNTs affects adsorption 


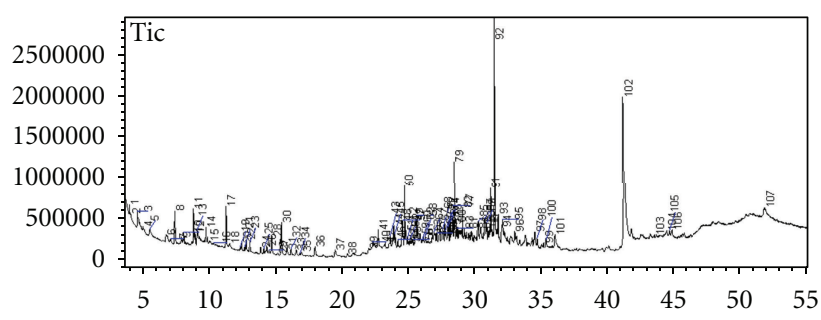

(a)

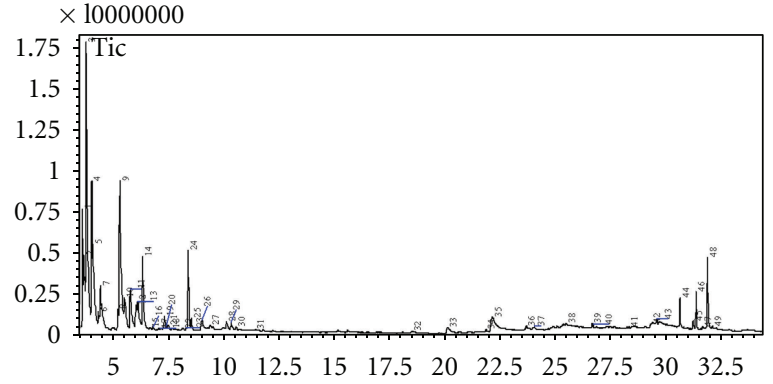

(b)

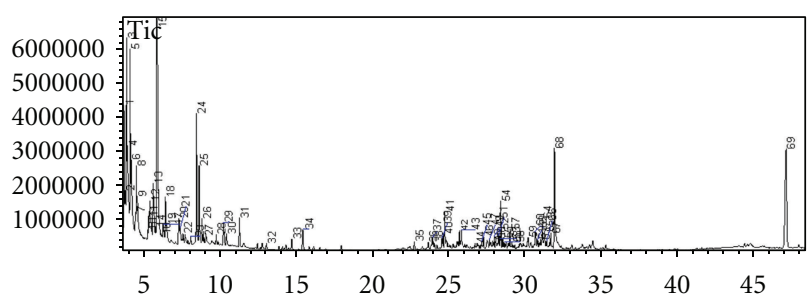

(c)

FIGURE 5: GC/MS spectra of the coking wastewater samples of (a) untreated, (b) treated by the MWCNT-ME for 120 min, (c) treated by the $\mathrm{IrSnSb} / \mathrm{Ti}$ electrode for $120 \mathrm{~min}$.

efficiency of single-ring aromatic organic pollutants with small molecular diameter in coking wastewater, which is consistent with the result in UV-vis spectrum. As the binder of the MWCNT-ME, infiltration of PTFE over the MWCNTs will decrease adsorption sites. In addition, only MWCNTs on the surface of MWCNT-ME can react with pollutants in the solution. Compared with the powder adsorption, media active adsorption sites on the MWCNT-ME were reduced. Adsorption ability is weakened, which makes electrochemical degradation be the determinant of concentration reduction of the pollutants.

3.3. Electrochemical Measurement. Electrochemical oxidation is a common way to abate phenol and Aniline. MWCNT-ME is expected to have good performance when used for electrochemical oxidation of phenol and aniline. Figure 3(a) shows the cyclic voltammeter of the MWCNT$\mathrm{ME}$ in base solution and solution with trace phenol in the potential range from $-0.2 \mathrm{~V}$ to $1.4 \mathrm{~V}$. Compared with base solution, the CV curve of phenol solution has an obvious oxidation peak on the potential of about $0.95 \mathrm{~V}$. This oxidation peak is corresponding to electrocatalytic oxidation of phenol and the current at oxidation peak reaches $0.18 \mathrm{~A}$. The same trend was observed in electrochemical oxidation of aniline (Figure 3(b)). Compared with base solution, the oxidation peak with current of $0.12 \mathrm{~A}$ on the potential of about $0.45 \mathrm{~V}$ occurred on the CV curve in the potential range from $-0.2 \mathrm{~V}$ to $1.4 \mathrm{~V}$. Above trend indicates that oxidation of phenol and aniline are carried on obviously, which means that MWCNTME can improve the sensitivity and electrocatalytic activity to oxidation of phenol and aniline in coking wastewater.

3.4. Electrochemical Degradation. The UV-vis spectrum during electrochemical oxidation of coking wastewater by
MWCNT-ME was showed in Figure 4. An absorption band from $200 \mathrm{~nm}$ to $400 \mathrm{~nm}$ was observed. An absorption peak occurred in the range of 190 250 nm. There exist B absorption band and $\mathrm{E}$ absorption band of unsaturated structures of organic pollutants in the UV-vis region. Maximum wavelength of $\mathrm{E}$ absorption band of single-ring aromatic compounds (such as phenol and aniline) is in the range of $200 \mathrm{~nm} \sim 250 \mathrm{~nm}$. The maximum wavelength of B absorption band of single-ring aromatic compounds is about $275 \mathrm{~nm}$. The maximum wavelength of the $\mathrm{E}$ absorption band and $\mathrm{B}$ absorption band of polycyclic aromatic hydrocarbons and heterocyclic compounds (such as naphthalene, acridine, quinoline, and indole) is about $275 \mathrm{~nm}$ and in the range of $300 \sim 375 \mathrm{~nm}$, respectively. According to the above policies, the adsorption peak in the range of $200 \sim 250 \mathrm{~nm}$ is formed by $\mathrm{E}$ adsorption band of single-ring aromatic compounds in the coking wastewater. Weak adsorption peak in the range of $250 \sim 300 \mathrm{~nm}$ is formed by B adsorption band of single-ring aromatic compounds and $\mathrm{E}$ adsorption band of polycyclic aromatic hydrocarbons and heterocyclic organic compounds in the coking wastewater. The adsorption band in the range of $300 \sim 370 \mathrm{~nm}$ is formed by B adsorption band of polycyclic aromatic hydrocarbons and heterocyclic organic compounds. With the degradation, the adsorption band became weaker and weaker. The concentration of the organic pollutants decreased. In the first 30 mins, adsorption bands $(200 \sim 250 \mathrm{~nm}$ and $250 \sim 300 \mathrm{~nm})$ decreased significantly, which was consistent with the electrochemical degradation of the phenol and aniline. At 60 mins, the adsorption band in $250 \sim 300 \mathrm{~nm}$ fall with flat trend. When the time reached $120 \mathrm{~min}$, the significant downward trend of the entire absorption band cannot be found. From the overall trend, different types of organic pollutants in the coking wastewater have been degraded to varying degrees. 
TABLE 1: COD of coking wastewater treated by IrSnSb/Ti electrode and MWCNT-ME.

\begin{tabular}{lcc}
\hline & IrSnSb/Ti & MWCNT-ME \\
\hline $\begin{array}{l}\text { COD (after tow-hour } \\
\text { electrochemical degradation) }\end{array}$ & 93 & 71 \\
Degradation efficiency (\%) & 35.9 & 51 \\
\hline
\end{tabular}

Figure 5 gives the GC/MS spectra of coking wastewater samples untreated and treated by $\mathrm{IrSnSb} / \mathrm{Ti}$ electrode and MWCNT-ME. One hundred and seven kinds of organic compounds were detected in the untreated coking wastewater. Forty kinds of organic compounds were removed by electrochemical degradation with $\mathrm{IrSnSb} / \mathrm{Ti}$ electrode. Fiftyeight kinds of organic compounds were removed by electrochemical degradation with MWCNT-ME. In addition, preliminary quantitative analysis of COD test was carried out for two electrodes after two-hour electrochemical degradation. Degradation efficiencies were shown in Table 1. The result shows that the MWCNT-ME has better degradation reactivity than $\mathrm{IrSnSb} / \mathrm{Ti}$ electrode.

\section{Conclusion}

An MWCNT-ME was made. Basic electrode configuration of the MWCNT-ME is randomly dispersed MWCNTs in PTFE dropped on the surface of a Ti conducting support macroelectrode. Adsorption activity of MWCNT-ME was proved to be weaker than powder adsorption media. The removal of refractory organic pollutants in coking wastewater mainly depends on electrochemical degradation occurred on MWCNT-ME. The CV curves for phenol and aniline have obvious oxidation and reduction peaks which reflect electrochemical reactivity of MWCNT-ME for some refractory organic pollutants. Such reactivity is furthermore reflected in the concentration decrease of the organic pollutants expressed in the UV-vis spectrum. The COD removal and change of organic pollutant species showed that MWCNT$\mathrm{ME}$ has better performance than $\mathrm{IrSnSb} / \mathrm{Ti}$ electrode on degradation efficiency. MWCNT-ME could be applied to process degradation of refractory organic pollutant in coking wastewater.

\section{Acknowledgment}

The financial support of Shanghai Science and Technology Cooperation Foundation (08230707200 and 0952nm06300) and Natural Science Foundation of China (91023029) is gratefully acknowledged.

\section{References}

[1] Y. H. Cui, X. Y. Li, and G. Chen, "Electrochemical degradation of bisphenol A on different anodes," Water Research, vol. 43, no. 7, pp. 1968-1976, 2009.

[2] C. A. Martínez-Huitle, A. De Battisti, S. Ferro, S. Reyna, M. Cerro-López, and M. A. Quiro, "Removal of the pesticide methamidophos from aqueous solutions by electrooxidation using
$\mathrm{Pb} / \mathrm{PbO}_{2}, \mathrm{Ti} / \mathrm{SnO}_{2}$, and $\mathrm{Si} / \mathrm{BDD}$ electrodes," Environmental Science and Technology, vol. 42, no. 18, pp. 6929-6935, 2008.

[3] F. Montilla, E. Morallón, and J. L. Vázquez, "Evaluation of the electrocatalytic activity of antimony-doped tin dioxide anodes toward the oxidation of phenol in aqueous solutions," Journal of the Electrochemical Society, vol. 152, no. 10, pp. B421-B427, 2005.

[4] C. Tan, B. Xiang, Y. Li, J. Fang, and M. Huang, "Preparation and characteristics of a nano- $\mathrm{PbO}_{2}$ anode for organic wastewater treatment," Chemical Engineering Journal, vol. 166, no. 1, pp. 15-21, 2011.

[5] R. Saito, M. Fujita, G. Dresselhaus, and M. S. Dresselhaus, "Electronic structure of chiral graphene tubules," Applied Physics Letters, vol. 60, no. 18, pp. 2204-2206, 1992.

[6] F. Wu, G. Zhao, and X. Wei, "Electrocatalytic activity of multiwalled carbon nanotubes modified electrode for p-benzenediol," Fenxi Huaxue, vol. 32, no. 8, pp. 1057-1060, 2004.

[7] L. C. Chen, Y. C. Ho, W. S. Guo, C. M. Huang, and T. C. Pan, "Enhanced visible light-induced photoelectrocatalytic degradation of phenol by carbon nanotube-doped $\mathrm{TiO}_{2}$ electrodes," Electrochimica Acta, vol. 54, no. 15, pp. 3884-3891, 2009.

[8] G. Busca, S. Berardinelli, C. Resini, and L. Arrighi, "Technologies for the removal of phenol from fluid streams: a short review of recent developments," Journal of Hazardous Materials, vol. 160, no. 2-3, pp. 265-288, 2008.

[9] Y. Show and H. Itabashi, "Electrically conductive material made from CNT and PTFE," Diamond and Related Materials, vol. 17, no. 4-5, pp. 602-605, 2008. 

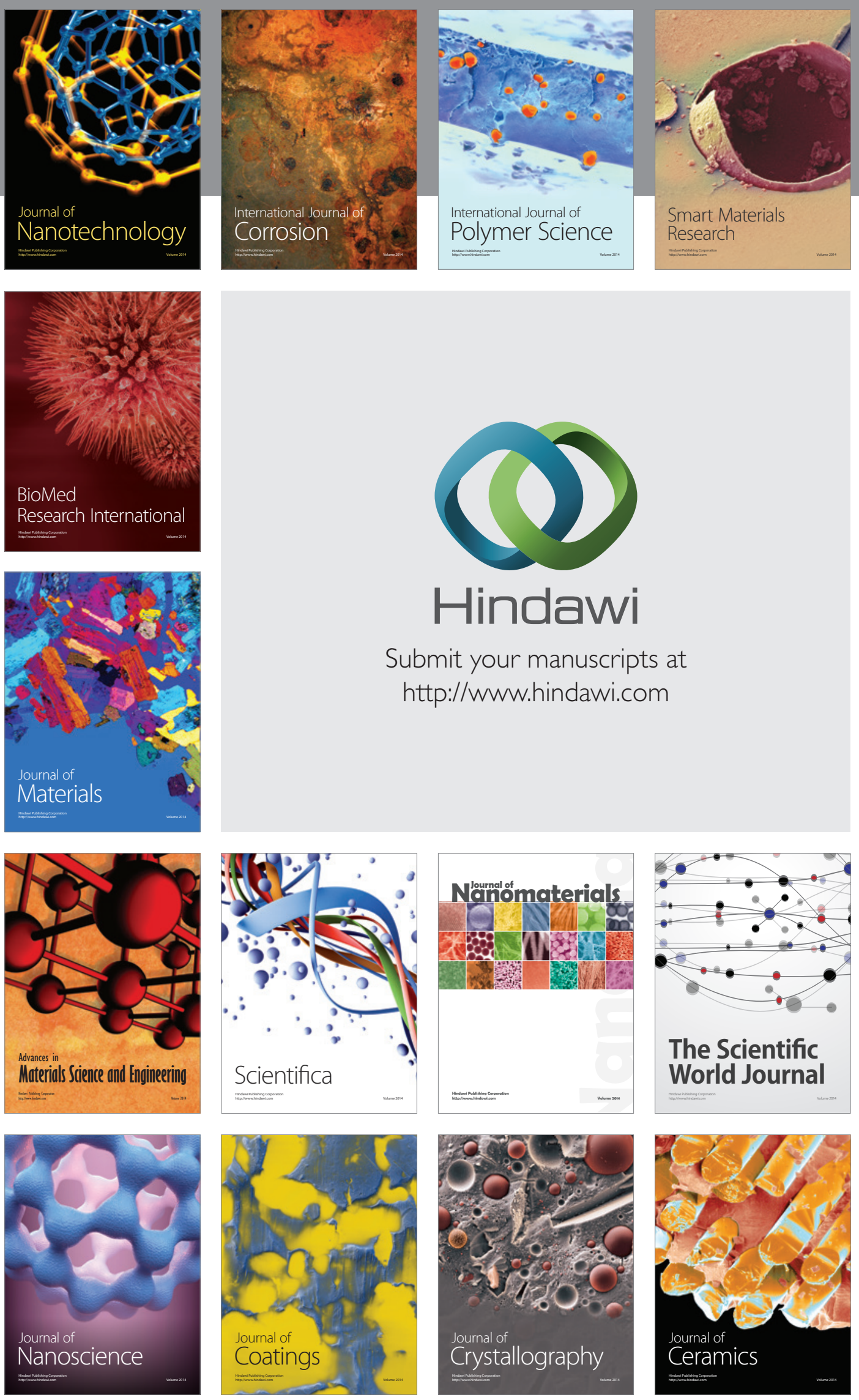

The Scientific World Journal

Submit your manuscripts at

http://www.hindawi.com

\section{World Journal}

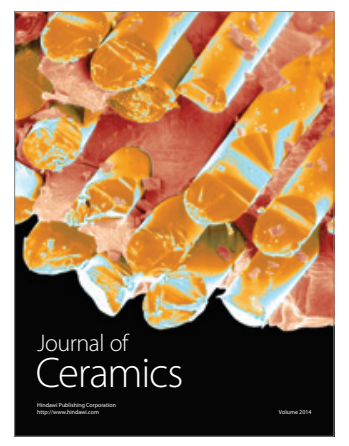

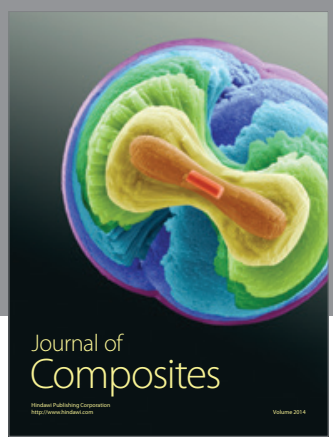
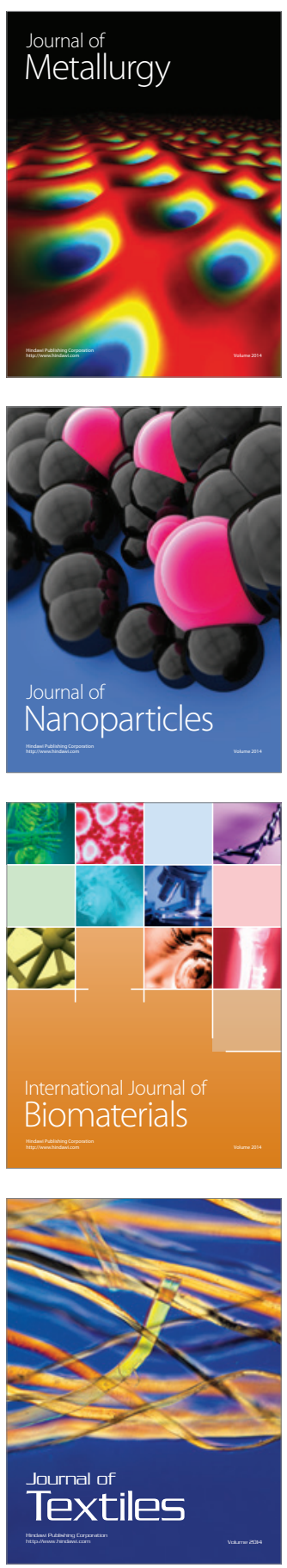